\title{
A Novel Class of Inhibitors of Peptide Deformylase Discovered Through High Throughput Screening and \\ Virtual Ligand Screening
}

Michael H. Howard, ${ }^{* \dagger}$ Teodorica Cenizal, ${ }^{\dagger}$ Steven Gutteridge, ${ }^{\dagger}$ Wayne S. Hanna, ${ }^{\dagger}$ Yong Tao, ${ }^{\dagger}$ Maxim Totrov, ${ }^{*}$ Vernon A. Wittenbach ${ }^{\dagger}$ and Ya-jun Zheng $^{\dagger}$

${ }^{\dagger}$ DuPont Crop Protection, Stine-Haskell Research Center, 1094 Elkton Road, Newark, Delaware 19711 and ${ }^{\ddagger}$ Molsoft LLC, 3366 North Torrey Pines Court, Suite 300, La Jolla, California 92037

michael.h.howard-1@usa.dupont.com

\section{JM049222O}

\section{SUPPORTING INFORMATION}

Experimental procedures for new compounds..................................... 2

Analytical data for compounds from corporate collection and vendors.................S10

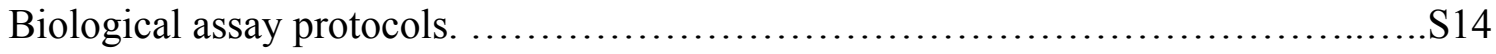

Virtual Ligand Screening: additional details...................................... 15

References for Supporting Information.......................................... 
JM049222O - Supporting Information

General Procedures. Common reagents were purchased from commercial suppliers. 4oxo-2-thioxo-3-thiazolidineacetic acid was purchased from Aldrich (Milwaukee, WI) and 4-oxo-2-thioxo-3-thiazolidinepropanoic acid was purchased from TCI-America (Portland, OR). All reactions were carried out under an inert nitrogen atmosphere with dry solvents, under anhydrous conditions, unless otherwise stated. Organic solutions were dried over anhydrous $\mathrm{Na}_{2} \mathrm{SO}_{4}$ before being concentrated. TLC was performed on silica gel 60 F254 (Merck) with detection by UV light. Column chromatography was performed on silica gel (60-120 micron). Analytical reversed-phase HPLC was performed on a BDS C-18 column $(250 \mathrm{~mm}$ x $4.6 \mathrm{~mm})$ eluted with a gradient of $\mathrm{MeCN}$ in $\mathrm{H}_{2} \mathrm{O}$ containing $0.1 \%$ TFA (flow rate of $0.50 \mathrm{~mL} / \mathrm{min}$, with a detection range of 200-400 $\mathrm{nm}$ using maximum chromatogram). ${ }^{1} \mathrm{H}$ and ${ }^{13} \mathrm{C}$ spectra were recorded at 300 or at 400 $\mathrm{MHz}$ and 75 or at $100 \mathrm{MHz}$, respectively, for solutions in $\mathrm{CDCl}_{3}$ [residual $\mathrm{CHCl}_{3}\left(\delta_{\mathrm{H}}\right.$ $7.26 \mathrm{ppm})$ or $\mathrm{CDCl}_{3}\left(\delta_{\mathrm{C}} 77.0 \mathrm{ppm}\right)$ as internal standard.

4-Oxo-2-thioxo-3-thiazolidinehexanoic acid (17e) was prepared according to the literature procedure..$^{1}$ The corresponding butanoic $(\mathbf{1 7 c})$, pentanoic (17d), heptanoic (17f) and octanoic acids (17g) were prepared in the same manner from the appropriate amino acids and their characterization data is provided, below.

4-Oxo-2-thioxo-3-thiazolidinebutanoic acid (17c): 4-aminobutanoic acid (10.31 g, 100 $\mathrm{mmol}), 22 \%$ aq. $\mathrm{KOH}(50 \mathrm{~mL})$, carbon disulfide $(9.7 \mathrm{~mL}, 100 \mathrm{mmol})$, chloroacetic acid (9.45 g, $100 \mathrm{mmol})$, and conc. $\mathrm{H}_{2} \mathrm{SO}_{4}(32 \mathrm{~mL}, 180 \mathrm{mmol})$ afforded the corresponding butanoic acid (14.78 g, $67 \%)$ as an off-white solid: mp $124-127{ }^{\circ} \mathrm{C} ;{ }^{1} \mathrm{H}$ NMR $(300$ 
JM049222O - Supporting Information

$\left.\mathrm{MHz}, \mathrm{CDCl}_{3}\right) \delta 1.99-2.06(\mathrm{~m}, 2 \mathrm{H}), 2.45(\mathrm{t}, 2 \mathrm{H}, J=7.2), 3.98(\mathrm{~s}, 2 \mathrm{H}), 4.09(\mathrm{t}, 2 \mathrm{H}, J=$ 7.2); MS AP(-) m/z $218.01(\mathrm{M}-\mathrm{H})^{-}$. Anal. Calcd for $\mathrm{C}_{7} \mathrm{H}_{9} \mathrm{NO}_{3} \mathrm{~S}_{2}$ : C, 38.34; H, 4.14;

N, 6.39. Found: C, 38.64; H, 3.98; N, 6.32.

4-Oxo-2-thioxo-3-thiazolidinepentanoic acid (17d): 5-Aminopentanoic acid (15.36 g, $100 \mathrm{mmol}), 22 \%$ aq. $\mathrm{KOH}(50 \mathrm{~mL})$, carbon disulfide $(9.7 \mathrm{~mL}, 100 \mathrm{mmol})$, chloroacetic acid $(9.45 \mathrm{~g}, 100 \mathrm{mmol})$ and conc. $\mathrm{H}_{2} \mathrm{SO}_{4}(32 \mathrm{~mL}, 180 \mathrm{mmol})$ afforded the corresponding pentanoic acid $(9.44 \mathrm{~g}, 41 \%)$ as an off white solid: $\mathrm{mp} 117-119{ }^{\circ} \mathrm{C} ;{ }^{1} \mathrm{H}$ NMR $(300$ $\left.\mathrm{MHz}, \mathrm{CDCl}_{3}\right) \delta 1.62-1.81(\mathrm{~m}, 4 \mathrm{H}), 2.41(\mathrm{t}, 2 \mathrm{H}, J=6.9), 3.93-4.08(\mathrm{~m}, 4 \mathrm{H}) ; \operatorname{MS}$ AP(-) m/z 232.02 (M-H) ${ }^{-}$. Anal. Calcd for $\mathrm{C}_{8} \mathrm{H}_{11} \mathrm{NO}_{3} \mathrm{~S}_{2}$ : C, 41.19; H, 4.75; N, 6.00; S, 27.49. Found: C, 40.98; H, 4.65; N, 5.90; S, 27.70.

4-Oxo-2-thioxo-3-thiazolidineheptanoic acid (17f): 7-aminoheptanoic acid (1.0 g, 6.9 mmol), aq. $\mathrm{KOH}(0.78 \mathrm{~g}, 13.7 \mathrm{mmol})$, carbon disulfide $(0.53 \mathrm{~g}, 6.9 \mathrm{mmol})$, chloroacetic acid $(0.65 \mathrm{~g}, 6.9 \mathrm{mmol})$ and conc. $\mathrm{H}_{2} \mathrm{SO}_{4}(1.22 \mathrm{~g}, 12.4 \mathrm{mmol})$ afforded the corresponding heptanoic acid $(0.55 \mathrm{~g}, 30 \%)$ as an off-white solid: $\mathrm{mp} \quad 74-76{ }^{\circ} \mathrm{C} ;{ }^{1} \mathrm{H}$ NMR $(300 \mathrm{MHz}$, $\left.\mathrm{CDCl}_{3}\right) \delta 1.27-1.46(\mathrm{~m}, 4 \mathrm{H}), 1.56-1.72(\mathrm{~m}, 4 \mathrm{H}), 2.35(\mathrm{t}, 2 \mathrm{H}, J=7.4), 3.91-4.03(\mathrm{~m}, 4 \mathrm{H})$ ${ }^{13} \mathrm{C} \mathrm{NMR}\left(75 \mathrm{MHz}, \mathrm{CDCl}_{3}\right) \delta 24.4,26.3,26.5,28.5,33.8,35.3,44.6,173.9,179.7,201.2$; MS AP(-) m/z $260.11(\mathrm{M}-\mathrm{H})^{-}$.

4-Oxo-2-thioxo-3-thiazolidineoctanoic acid (17g): 8-Aminooctanoic acid (1.0 g, 6.3 $\mathrm{mmol})$, aq. $\mathrm{KOH}(0.71 \mathrm{~g}, 12.5 \mathrm{mmol})$, carbon disulfide $(0.48 \mathrm{~g}, 6.3 \mathrm{mmol})$, chloroacetic acid $(0.59 \mathrm{~g}, 6.3 \mathrm{mmol})$ and conc. $\mathrm{H}_{2} \mathrm{SO}_{4}(1.2 \mathrm{~g}, 11.3 \mathrm{mmol})$ afforded the corresponding 
JM049222O - Supporting Information

octanoic acid (0.50 g, 29\%) as a yellow solid: $\mathrm{mp} 82-84{ }^{\circ} \mathrm{C} ;{ }^{1} \mathrm{H}$ NMR $(300 \mathrm{MHz}$, $\left.\mathrm{CDCl}_{3}\right) \delta 1.29-139(\mathrm{~m}, 6 \mathrm{H}), 1.56-1.70(\mathrm{~m}, 4 \mathrm{H}), 2.35(\mathrm{t}, 2 \mathrm{H}, J=7.3), 3.93-4.00(\mathrm{~m}, 4 \mathrm{H})$

${ }^{13} \mathrm{C}$ NMR $\left(75 \mathrm{MHz}, \mathrm{CDCl}_{3}\right) \delta 24.5,26.5,26.6,28.7,28.8,33.9,35.3,44.7,173.9,179.7$, 201.2; MS AP(-) m/z 274.11(M-H) ${ }^{-}$. Anal. Calcd for $\mathrm{C}_{11} \mathrm{H}_{17} \mathrm{NO}_{3} \mathrm{~S}_{2}$ : C, 47.98; H, 6.22; N, 5.09; S, 23.29. Found: C, 48.17; H, 6.06; N, 4.93; S, 22.94.

\section{5-[(2,5-Dimethyl-1-phenyl-1H-pyrrol-3-yl)methylene]-4-oxo-2-thioxo-3-}

thiazolidinehexanoic acid (2). Ethylenediamine diacetate (2.18 g, $12.0 \mathrm{mmol})$ and 2,5dimethyl-1-phenylpyrrole-3-carboxaldehyde (2.41 g, $12.0 \mathrm{mmol})$, was added to stirring solution of 4-oxo-2-thioxo-3-thiazolidinehexanoic acid (17e) (3.00 g, $12.0 \mathrm{mmol})$ in methanol (45 mL) at rt. After $1 \mathrm{~h}$, a yellow precipitate formed. Stirring was continued overnight at room temperature. The precipitate was filtered, washed with water and dried under high vacuum to give $2(4.12 \mathrm{~g}, 79 \%)$ as a bright yellow solid: $\mathrm{mp} 184-186{ }^{\circ} \mathrm{C} ;{ }^{1} \mathrm{H}$ NMR (300 MHz, $\left.\mathrm{CDCl}_{3}\right) \delta 1.35-1.52(\mathrm{~m}, 2 \mathrm{H}), 1.64-1.83(\mathrm{~m}, 4 \mathrm{H}), 2.03(\mathrm{~s}, 3 \mathrm{H}), 2.18(\mathrm{~s}$, $3 \mathrm{H}), 2.38(\mathrm{t}, 2 \mathrm{H}, J=7.5), 4.12(\mathrm{t}, 2 \mathrm{H}, J=7.6), 6.20(\mathrm{~s}, 1 \mathrm{H}), 7.18-7.21(\mathrm{~m}, 2 \mathrm{H}), 7.45-7.56$ (m, 3H), $7.75(\mathrm{~s}, 1 \mathrm{H}) ;{ }^{13} \mathrm{C} \mathrm{NMR}\left(75 \mathrm{MHz}, \mathrm{CDCl}_{3}\right) \delta 11.1,12.8,24.2,26.2,26.6,33.8$, $44.1,105.8,114.8,116.4,127.8,127.8,128.9,129.6,132.6,137.2,137.6,168.0,179.3$, 193.5; $\mathrm{MS} \mathrm{AP}(-) m / z 427.12(\mathrm{M}-\mathrm{H})^{-}$. Anal. Calcd for $\mathrm{C}_{22} \mathrm{H}_{24} \mathrm{~N}_{2} \mathrm{O}_{3} \mathrm{~S}_{2}$ : C, 61.66; H, 5.64; N, 6.54; S, 14.96. Found: C, 61.30; H, 5.43; N, 6.48; S, 15.13.

\section{5-[(2,5-Dimethyl-1-phenyl-1H-pyrrol-3-yl)methylene]-4-oxo-2-thioxo-3-}

thiazolidinebutanoic acid (18). Following the same procedure as for 2, ethylenediamine diacetate (0.252 g, $1.14 \mathrm{mmol}), 2,5$-dimethyl-1-phenylpyrrole-3-carboxaldehyde $(0.227$ g, $1.14 \mathrm{mmol}$ ) and 4-oxo-2-thioxo-3-thiazolidinebutanoic acid (0.250 g, $1.14 \mathrm{mmol})$ 
JM049222O - Supporting Information

afforded 18 (290 mg, 63\%) as a yellow solid: $\mathrm{mp} 87-89{ }^{\circ} \mathrm{C}$; ${ }^{1} \mathrm{H}$ NMR $(300 \mathrm{MHz}$, $\left.\mathrm{CDCl}_{3}\right) \delta 2.03(\mathrm{~s}, 3 \mathrm{H}), 2.04-2.15(\mathrm{~m}, 2 \mathrm{H}), 2.18(\mathrm{~s}, 3 \mathrm{H}), 2.46(\mathrm{t}, 2 \mathrm{H}, J=7.5), 4.22(\mathrm{t}, 2 \mathrm{H}, J$ $=6.8), 6.20(\mathrm{~s}, 1 \mathrm{H}), 7.16-7.23(\mathrm{~m}, 2 \mathrm{H}), 7.45-7.57(\mathrm{~m}, 3 \mathrm{H}), 7.76(\mathrm{~s}, 1 \mathrm{H}) ; \operatorname{MS~AP}(+) \mathrm{m} / \mathrm{z}$ $401.03(\mathrm{M}+\mathrm{H})^{+}$. Anal. Calcd for $\mathrm{C}_{20} \mathrm{H}_{20} \mathrm{~N}_{2} \mathrm{O}_{3} \mathrm{~S}_{2}$ : C, 59.98; H, 5.03; N, 6.99; S, 16.01. Found: C, 60.11; H, 5.02; N, 6.83; S, 15.85 .

\section{5-[(2,5-Dimethyl-1-phenyl-1H-pyrrol-3-yl)methylene]-4-oxo-2-thioxo-3-}

thiazolidinepentanoic acid (19). Following the same procedure as for 2, ethylenediamine diacetate $(2.31 \mathrm{~g}, 12.8 \mathrm{mmol}), 2,5$-dimethyl-1-phenylpyrrole-3carboxaldehyde (2.56 g, $12.8 \mathrm{mmol})$ and 4-oxo-2-thioxo-3-thiazolidinepentanoic acid (3.00 g, $12.8 \mathrm{mmol})$ afforded $19(3.55 \mathrm{~g}, 67 \%)$ as an orange solid: $\mathrm{mp} 88-90{ }^{\circ} \mathrm{C} ;{ }^{1} \mathrm{H}$ NMR $\left(300 \mathrm{MHz}, \mathrm{CDCl}_{3}\right) \delta 1.67-1.87(\mathrm{~m}, 4 \mathrm{H}), 2.03(\mathrm{~s}, 3 \mathrm{H}), 2.18(\mathrm{~s}, 3 \mathrm{H}), 2.44(\mathrm{t}, 2 \mathrm{H}, J=$ 7.2), $4.15(\mathrm{t}, 2 \mathrm{H}, J=7.2), 6.20(\mathrm{~s}, 1 \mathrm{H}), 7.18-7.23(\mathrm{~m}, 2 \mathrm{H}), 7.47-7.53(\mathrm{~m}, 3 \mathrm{H}), 7.75(\mathrm{~s}$, $1 \mathrm{H}) ;{ }^{13} \mathrm{C} \mathrm{NMR} \delta 24.2,25.2(2 \mathrm{C}), 25.6,26.2,26.6,31.2(2 \mathrm{C}), 33.8,41.6,44.2,125.0$, 142.9, 166.6, 179.7, 194.0; $\mathrm{MS} \mathrm{AP}(+) m / z$ 415.15 $(\mathrm{M}+\mathrm{H})^{+}$. Anal. Calcd for $\mathrm{C}_{21} \mathrm{H}_{22} \mathrm{~N}_{2} \mathrm{O}_{3} \mathrm{~S}_{2}:$ C, 60.85; H, 5.35; N, 6.76; S, 15.47. Found: C, 60.90; H, 5.31; N, $6.76 ; \mathrm{S}, 15.50$.

\section{5-[(2,5-Dimethyl-1-phenyl-1H-pyrrol-3-yl)methylene]-4-oxo-2-thioxo-3-}

thiazolidineheptanoic acid (20): Following the same procedure as for 2,

ethylenediamine diacetate (1.09 g, $6.7 \mathrm{mmol}), 2$,5-dimethyl-1-phenylpyrrole-3carboxaldehyde (1.33 g, $6.7 \mathrm{mmol})$ and 4-oxo-2-thioxo-3-thiazolidineheptanoic acid $(1.75 \mathrm{~g}, 6.7 \mathrm{mmol})$ afforded $20(2.1 \mathrm{~g}, 71 \%)$ as an orange solid: $\mathrm{mp} 162-163{ }^{\circ} \mathrm{C} ;{ }^{1} \mathrm{H}$ 
JM049222O - Supporting Information

NMR (300 MHz, $\left.\mathrm{CDCl}_{3}\right) \delta$ 1.35-1.47 (m, 4H), 1.64-1.74 (m, 4H), $2.03(\mathrm{~s}, 3 \mathrm{H}), 2.18(\mathrm{~s}$, $3 \mathrm{H}), 2.36(\mathrm{t}, 2 \mathrm{H}, J=7.4), 4.12(\mathrm{t}, 2 \mathrm{H}, J=7.6), 6.21(\mathrm{~s}, 1 \mathrm{H}), 7.20(\mathrm{~m}, 2 \mathrm{H}), 7.51(\mathrm{~m}, 3 \mathrm{H})$ and $7.75(\mathrm{~s}, 1 \mathrm{H})$; MS (+ ve mode): $\mathrm{m} / \mathrm{z} 443.2(\mathrm{M}+\mathrm{H})^{+}$; HPLC purity $91 \%(\mathrm{BDS})$.

\section{5-[(2,5-Dimethyl-1-phenyl-1H-pyrrol-3-yl)methylene]-4-oxo-2-thioxo-3-}

thiazolidineoctanoic acid (21): Following the same procedure as for 2, ethylenediamine diacetate (590 mg, $3.6 \mathrm{mmol}$ ), 2,5-dimethyl-1-phenylpyrrole-3-carboxaldehyde (720 mg, $3.6 \mathrm{mmol})$ and 4-oxo-2-thioxo-3-thiazolidineoctanoic acid (1.0 g, $3.6 \mathrm{mmol})$ afforded 21 (600 mg, 38\%) as an orange solid: $\mathrm{mp} 128-129{ }^{\circ} \mathrm{C} ;{ }^{1} \mathrm{H}$ NMR $\left(300 \mathrm{MHz}, \mathrm{CDCl}_{3}\right) \delta 1.38$ (m, 4H), $1.64(\mathrm{~m}, 6 \mathrm{H}), 2.03(\mathrm{~s}, 3 \mathrm{H}), 2.18(\mathrm{~s}, 3 \mathrm{H}), 2.36(\mathrm{t}, 2 \mathrm{H}, J=7.4), 4.12(\mathrm{t}, 2 \mathrm{H}, J=$ 7.6), $6.21(\mathrm{~s}, 1 \mathrm{H}), 7.20(\mathrm{~m}, 2 \mathrm{H}), 7.51(\mathrm{~m}, 3 \mathrm{H})$ and $7.75(\mathrm{~s}, 1 \mathrm{H}) ;{ }^{13} \mathrm{C}$ NMR $(75 \mathrm{MHz}$, $\left.\mathrm{CDCl}_{3}\right) \delta 11.1,12.7,24.5,26.5,26.8,28.8(2 \mathrm{C}), 33.9,44.4,105.8,115.0,116.4,127.8$ (2C), 128.9, 129.6, 137.3, 137.5, 168.0, 179.5 and 193.5; MS (+ ve mode): $\mathrm{m} / \mathrm{z} 457.2$ $(\mathrm{M}+\mathrm{H})^{+}$; HPLC purity $85 \%$. Anal. Calcd for $\mathrm{C}_{24} \mathrm{H}_{28} \mathrm{~N}_{2} \mathrm{O}_{3} \mathrm{~S}_{2}: \mathrm{C}, 63.13 ; \mathrm{H}, 6.18 ; \mathrm{N}, 6.13$; S, 14.04. Found: C, 62.98; H, 6.03; N, 6.21; S, 14.05 .

\section{N-Hydroxy-5-[(2,5-dimethyl-1-phenyl-1H-pyrrol-3-yl)methylene]-4-oxo-2-thioxo-3-}

thiazolidinehexamide (22). $O$-(tetrahydro-2H-pyran-2-yl)hydroxylamine (160 mg, 1.4 mmol) was added to a solution of the acid $2(500 \mathrm{mg}, 1.2 \mathrm{mmol})$ in dry dichloromethane $(10 \mathrm{~mL})$ at $\mathrm{rt}$ and cooled it to $0{ }^{\circ} \mathrm{C}$. To this cold solution was added 1hydroxybenzotriazole hydrate (190 mg, $1.4 \mathrm{mmol})$ and 1-(3-dimethylaminopropyl)-3ethylcarbodiimide hydrochloride $(270 \mathrm{mg}, 1.4 \mathrm{mmol})$ sequentially. The mixture was then allowed to warm to rt and stir overnight. The reaction mixture was diluted with 
JM049222O - Supporting Information

dichloromethane $(20 \mathrm{~mL})$ and the dichloromethane layer was washed with water $(2 \mathrm{x} 10$ $\mathrm{mL})$, brine $(10 \mathrm{~mL})$ and dried $\left(\mathrm{Na}_{2} \mathrm{SO}_{4}\right)$, filtered and concentrated. The crude material so obtained was taken directly to the next step without further purification.

p-Toluenesulfonic acid (20 mg, $0.12 \mathrm{mmol}$ ) was added to a solution of the crude $O$-THP hydroxamate in dry $\mathrm{MeOH}(10 \mathrm{~mL})$ and stirred at $\mathrm{rt}$ for $2 \mathrm{~h}$, at which point the reaction was judged to be complete as indicated by formation of an $\mathrm{FeCl}_{3}$ active spot on TLC. The reaction was quenched by adding water $(30 \mathrm{~mL})$ and extracted with ethyl acetate $(3 \mathrm{x} 15$ $\mathrm{mL})$. Ethyl acetate layer was washed with water $(2 \times 15 \mathrm{~mL})$ and brine $(15 \mathrm{~mL})$, then dried $\left(\mathrm{Na}_{2} \mathrm{SO}_{4}\right)$, filtered and concentrated. The crude compound was purified by column chromatography (silica gel, $\mathrm{CHCl}_{3} / \mathrm{MeOH}$ ) to obtain 22 in pure form as yellow solid (310 mg, 60\%). mp $110-113{ }^{\circ} \mathrm{C} ;{ }^{1} \mathrm{H} \mathrm{NMR}\left(400 \mathrm{MHz}, \mathrm{CDCl}_{3}\right) \delta 1.42(\mathrm{~m}, 2 \mathrm{H}), 1.77(\mathrm{~m}$, 4H), $2.04(\mathrm{~s}, 3 \mathrm{H}), 2.19(\mathrm{~s}, 3 \mathrm{H}), 2.23(\mathrm{~m}, 2 \mathrm{H}), 4.12(\mathrm{t}, 2 \mathrm{H}, J=7.4), 6.21(\mathrm{~s}, 1 \mathrm{H}), 7.20(\mathrm{~m}$, 2H), $7.52(\mathrm{~m}, 3 \mathrm{H})$ and $7.76(\mathrm{~s}, 1 \mathrm{H}) ;{ }^{13} \mathrm{C} \mathrm{NMR}(75 \mathrm{MHz}) \delta 11.1,12.7,24.5,25.8,26.2$, $32.4,43.8,105.7,114.6,116.4,127.7,128.0,128.8,129.5,132.6,137.2,137.8,168.1$, 170.9, 193.4; MS (+ ve mode): m/z $444(\mathrm{M}+\mathrm{H})^{+}$; HPLC purity 92\% (BDS).

\section{5-Cyclohexylmethylene-4-oxo-2-thioxo-3-thiazolidinehexanoic acid (23): Following}

the same procedure as for 2 , ethylenediamine diacetate $(95.5 \mathrm{mg}, 0.53 \mathrm{mmol})$ and cyclohexanecarboxaldehyde (0.0554 mL, $0.53 \mathrm{mmol})$, afforded $23(75 \mathrm{mg}, 42 \%)$ as a yellow solid: $\mathrm{mp} 130-133{ }^{\circ} \mathrm{C}$; ${ }^{1} \mathrm{H}$ NMR $\left(300 \mathrm{MHz}, \mathrm{CDCl}_{3}\right) \delta 1.21-1.47(\mathrm{~m}, 6 \mathrm{H}), 1.63-$ $1.87(\mathrm{~m}, 10 \mathrm{H}), 2.07-2.21(\mathrm{~m}, 1 \mathrm{H}), 2.37(\mathrm{t}, 2 \mathrm{H}, J=7.4), 4.06(\mathrm{t}, 2 \mathrm{H}, J=7.6), 6.83(\mathrm{~d}, 1 \mathrm{H}$, $J=9.4) ; \operatorname{MS~AP}(-) m / z 340.10(\mathrm{M}-\mathrm{H})^{-}$. Anal. Calcd for $\mathrm{C}_{16} \mathrm{H}_{23} \mathrm{NO}_{3} \mathrm{~S}_{2}:$ C, 56.28; $\mathrm{H}$, 6.79; N, 4.10; S, 18.78. Found: C, 55.95; H, 6.56; N, 4.02; S, 18.46. 
JM049222O - Supporting Information

\section{5-[(2,5-Dimethyl-1-phenyl-1H-pyrrol-3-yl)methylene]-4-oxo-2-thioxo-3-}

thiazolidinehexanoic acid methyl ester (24). Iodomethane ( $0.168 \mathrm{~mL}, 0.52 \mathrm{mmol})$ was added to stirring mixture of $2(200 \mathrm{mg}, 0.47 \mathrm{mmol})$ and potassium carbonate (193 $\mathrm{mg}$, $1.4 \mathrm{mmol})$ in acetonitrile $(15 \mathrm{~mL})$ and allowed to react at $\mathrm{rt}$ overnight. The reaction mixture was filtered and the filtrate was diluted with ethyl acetate $(20 \mathrm{~mL})$. The organic phase was washed with $1 \mathrm{~N} \mathrm{NaOH}(3 \times 20 \mathrm{~mL})$ and brine $(1 \times 10 \mathrm{~mL})$, dried over $\mathrm{MgSO}_{4}$, filtered and concentrated to an orange oil. This oil was then purified by silica gel chromatography (10-40\% EtOAc /hexane gradient over 20 min.) and dried under high vacuum to afford the methyl ester $24(120 \mathrm{mg}, 58 \%)$ as a yellow solid: $\mathrm{mp} 124-126{ }^{\circ} \mathrm{C}$; ${ }^{1} \mathrm{H}$ NMR $\left(300 \mathrm{MHz}, \mathrm{CDCl}_{3}\right) \delta 1.33-1.49(\mathrm{~m}, 2 \mathrm{H}), 1.61-1.85(\mathrm{~m}, 4 \mathrm{H}), 2.03(\mathrm{~s}, 3 \mathrm{H}), 2.18$ (s, 3H), $2.33(\mathrm{t}, 2 \mathrm{H}, J=7.8), 4.12(\mathrm{t}, 2 \mathrm{H}, J=7.6), 6.21(\mathrm{~s}, 1 \mathrm{H}), 7.17-7.21(\mathrm{~m}, 2 \mathrm{H}), 7.44-$ $7.56(\mathrm{~m}, 3 \mathrm{H}), 7.47(\mathrm{~s}, 1 \mathrm{H}) ;{ }^{13} \mathrm{C} \mathrm{NMR}\left(75 \mathrm{MHz}, \mathrm{CDCl}_{3}\right) \delta 11.0,12.7,24.3,26.1,26.4$, $33.7,44.0,51.3,76.6,76.9,77.4,105.6,114.7,116.3,127.6(2 \mathrm{C}), 128.8,129.5,132.5$, 137.1, 137.4, 167.8, 173.8, 193.3; $\mathrm{MS} \mathrm{AP}(+) m / z 443.04(\mathrm{M}+\mathrm{H})^{+}$. Anal. Calcd for $\mathrm{C}_{23} \mathrm{H}_{26} \mathrm{~N}_{2} \mathrm{O}_{3} \mathrm{~S}_{2}$ : C, 62.42; H, 5.92; N, 6.38; S, 14.49. Found: C, 62.25; H, 5.70; N, $6.20 ; \mathrm{S}, 14.40$.

\section{5-[(2,5-Dimethyl-1-phenyl-1H-pyrrol-3-yl)methylene]-4-oxo-2-thioxo-3-}

thiazolidineacetic acid (25). 2,5-dimethyl-1-phenylpyrrole-3-carboxaldehyde (1.99 g, $10.0 \mathrm{mmol})$ and 4-oxo-2-thioxo-3-thiazolidineacetic acid (1.91 g, $10.0 \mathrm{mmol})$ was allowed to stir in $15 \mathrm{~mL}$ ethanol under reflux for $18 \mathrm{~h}$. The resulting precipitate was then filtered, washed with water and dried under high vacuum to afford 25 (2.49 g, 67\%) as an 
JM049222O - Supporting Information

orange solid: mp $248-250{ }^{\circ} \mathrm{C} ;{ }^{1} \mathrm{H}$ NMR $\left(300 \mathrm{MHz}, \mathrm{CDCl}_{3}\right) \delta 2.03(\mathrm{~s}, 3 \mathrm{H}), 2.17(\mathrm{~s}, 3 \mathrm{H})$, $4.83(\mathrm{~s}, 2 \mathrm{H}), 6.21(\mathrm{~s}, 1 \mathrm{H}), 7.20(\mathrm{~d}, 2 \mathrm{H}, J=8.1), 7.50-7.53(\mathrm{~m}, 3 \mathrm{H}), 7.8(\mathrm{~s}, 1 \mathrm{H}) ; \mathrm{MS}$ AP $(+) m / z 373.02(\mathrm{M}+\mathrm{H})^{+}, \mathrm{MS} \mathrm{AP}(-) m / z 371.04(\mathrm{M}-\mathrm{H})^{-}$.

\section{5-[(2,5-Dimethyl-1-phenyl-1H-pyrrol-3-yl)methylene]-4-oxo-2-thioxo-3-}

thiazolidinepropionic acid (26). Following the same procedure as for 25, 2,5-dimethyl1-phenylpyrrole-3-carboxaldehyde (1.99 g, $10.0 \mathrm{mmol})$ and 4-oxo-2-thioxo-3thiazolidinepropanoic acid (2.05 g, $10.0 \mathrm{mmol})$ afforded $26(2.21 \mathrm{~g}, 57 \%)$ as an orange solid: mp 242-244 ${ }^{\circ} \mathrm{C} ;{ }^{1} \mathrm{H}$ NMR $\left(300 \mathrm{MHz}, \mathrm{CDCl}_{3}\right) \delta 2.02(\mathrm{~s}, 3 \mathrm{H}), 2.18(\mathrm{~s}, 3 \mathrm{H}), 2.84(\mathrm{t}$, $2 \mathrm{H}, J=7.8), 4.44(\mathrm{t}, 2 \mathrm{H}, J=7.8), 6.20(\mathrm{~s}, 1 \mathrm{H}), 7.20(\mathrm{~d}, 2 \mathrm{H}, J=8.1), 7.50-7.53(\mathrm{~m}, 3 \mathrm{H})$, $7.78(\mathrm{~s}, 1 \mathrm{H}) ; \mathrm{MS} \mathrm{AP}(+) \mathrm{m} / \mathrm{z} 387.02(\mathrm{M}+\mathrm{H})^{+}$. 
JM049222O - Supporting Information

The other active compounds presented in Tables 1 and 2, from our corporate compound collection and from commercial sources (for the virtual ligand screening experiment), were characterized as follows:

5-[(1,3-Diphenyl-1H-pyrazol-4-yl)methylene]-4-oxo-2-thioxo-3-thiazolidinehexanoic acid (1). mp $174-176{ }^{\circ} \mathrm{C} ;{ }^{1} \mathrm{H}$ NMR (300 MHz, DMSO) $\delta 1.25-1.38$ (m, 2H), 1.47-1.70 $(\mathrm{m}, 4 \mathrm{H}), 2.19(\mathrm{t}, 2 \mathrm{H}, J=7.3), 4.03(\mathrm{t}, 2 \mathrm{H}, J=7.3), 7.42(\mathrm{t}, 1 \mathrm{H}, J=7.8), 7.54-7.61(\mathrm{~m}$, 6H), 7.64-7.68 (m, 2H), $8.06(\mathrm{~d}, 2 \mathrm{H}, J=7.7), 8.81(\mathrm{~s}, 1 \mathrm{H})$; MS AP(-) m/z 476.01 (M$\mathrm{H})^{-}$.

5-[(1-(4-Bromophenyl)-2,5-dimethyl-1H-pyrrol-3-yl)methylene]-4-oxo-2-thioxo-3thiazolidinehexanoic acid (3). $\mathrm{mp} 163-165{ }^{\circ} \mathrm{C} ;{ }^{1} \mathrm{H} \mathrm{NMR}\left(300 \mathrm{MHz}, \mathrm{CDCl}_{3}\right) \delta 1.38$ $1.50(\mathrm{~m}, 2 \mathrm{H}), 1.63-1.80(\mathrm{~m}, 4 \mathrm{H}), 2.03(\mathrm{~s}, 3 \mathrm{H}), 2.18(\mathrm{~s}, 3 \mathrm{H}), 2.30(\mathrm{t}, 2 \mathrm{H}, J=7.4), 4.11(\mathrm{t}$, $2 \mathrm{H}, J=7.8), 6.19(\mathrm{~s}, 1 \mathrm{H}), 7.09(\mathrm{~d}, 2 \mathrm{H}, J=8.6), 7.64(\mathrm{~d}, 2 \mathrm{H}, J=8.3), 7.72(\mathrm{~s}, 1 \mathrm{H}) ; \mathrm{MS}$ $\mathrm{AP}(+) m / z 508.88(\mathrm{M}+\mathrm{H})^{+}$.

5-[(5-Chloro-3-methyl-1-phenyl-1H-pyrazol-4-yl)methylene]-4-oxo-2-thioxo-3thiazolidinehexanoic acid (4). $\mathrm{mp} 115-116^{\circ} \mathrm{C} ;{ }^{1} \mathrm{H} \mathrm{NMR}\left(300 \mathrm{MHz}, \mathrm{CDCl}_{3}\right) \delta 1.39$ $1.50(\mathrm{~m}, 2 \mathrm{H}), 1.64-1.83(\mathrm{~m}, 4 \mathrm{H}), 2.39(\mathrm{t}, 2 \mathrm{H}, J=7.5), 2.44(\mathrm{~s}, 3 \mathrm{H}), 4.12(\mathrm{t}, 2 \mathrm{H}, J=7.5)$, 7.45-7.58 (m, 5H), $7.61(\mathrm{~s}, 1 \mathrm{H}) ; \operatorname{MS~AP}(+) \mathrm{m} / \mathrm{z} 451.94(\mathrm{M}+\mathrm{H})^{+}$.

5-[(3-(5-Methylfuran-2-yl)-1-phenyl-1H-pyrazol-4-yl)methylene]-4-oxo-2-thioxo-3thiazolidinehexanoic acid (5). $\mathrm{mp} 154-156^{\circ} \mathrm{C} ;{ }^{1} \mathrm{H} \mathrm{NMR}\left(300 \mathrm{MHz}, \mathrm{CDCl}_{3}\right) \delta 1.39$ - 
JM049222O - Supporting Information

$1.54(\mathrm{~m}, 2 \mathrm{H}), 1.83-1.66(\mathrm{~m}, 4 \mathrm{H}), 2.38(\mathrm{t}, 2 \mathrm{H}, J=7.5), 2.45(\mathrm{~s}, 3 \mathrm{H}), 4.13(\mathrm{t}, 2 \mathrm{H}, J=7.5)$ $6.16(\mathrm{~d}, 1 \mathrm{H}, J=3.6), 6.82(\mathrm{~d}, 1 \mathrm{H}, J=3.3), 7.35-7.42(\mathrm{~m}, 1 \mathrm{H}), 7.48-7.55(\mathrm{~m}, 2 \mathrm{H}), 7.77(\mathrm{~d}$, $2 \mathrm{H}, J=8.7), 8.07(\mathrm{~s}, 1 \mathrm{H}), 8.18(\mathrm{~s}, 1 \mathrm{H}) ; \operatorname{MS~AP}(+) \mathrm{m} / \mathrm{z} 482.00(\mathrm{M}+\mathrm{H})^{+}, \operatorname{MS} \operatorname{AP}(-) m / z$ $480.02(\mathrm{M}-\mathrm{H})^{-}$.

5-[(1-(2-Cyanoethyl)-3-methyl-1H-pyrazol-4-y)Imethylene]-4-oxo-2-thioxo-3thiazolidinehexanoic acid (6). $\mathrm{mp} 171-173{ }^{\circ} \mathrm{C} ;{ }^{1} \mathrm{H} \mathrm{NMR}\left(300 \mathrm{MHz}, \mathrm{CDCl}_{3}\right) \delta 1.33$ $1.52(\mathrm{~m}, 2 \mathrm{H}), 1.59-1.83(\mathrm{~m}, 4 \mathrm{H}), 2.33(\mathrm{t}, 2 \mathrm{H}, J=7.7), 2.39(\mathrm{~s}, 3 \mathrm{H}), 3.00(\mathrm{t}, 2 \mathrm{H}, J=7.1)$, $4.10(\mathrm{t}, 2 \mathrm{H}, J=7.6), 4.41(\mathrm{t}, 2 \mathrm{H}, J=6.5), 7.53(\mathrm{~s}, 1 \mathrm{H}), 7.72(\mathrm{~s}, 1 \mathrm{H}) ; \operatorname{MS} \operatorname{AP}(-) \mathrm{m} / \mathrm{z}$ $391.04(\mathrm{M}-\mathrm{H})^{-}$.

4-Oxo-5-[[1-phenyl-3-(2-thienyl)-1H-pyrazol-4-yl]methylene]-2-thioxo-3thiazolidinehexanoic acid (7). $\mathrm{mp} 163-164{ }^{\circ} \mathrm{C} ;{ }^{1} \mathrm{H} \mathrm{NMR}\left(300 \mathrm{MHz}, \mathrm{CDCl}_{3}\right) \delta 1.37-$ $1.50(\mathrm{~m}, 2 \mathrm{H}), 1.63-1.82(\mathrm{~m}, 4 \mathrm{H}), 2.31(\mathrm{t}, 2 \mathrm{H}, J=7.4), 4.12(\mathrm{t}, 2 \mathrm{H}, J=7.3), 7.19(\mathrm{dd}, 1 \mathrm{H}$, $J=3.6,5.1), 7.37-7.56(\mathrm{~m}, 5 \mathrm{H}), 7.81(\mathrm{~d}, 2 \mathrm{H}, J=7.5), 7.90(\mathrm{~s}, 1 \mathrm{H}), 8.16(\mathrm{~s}, 1 \mathrm{H})$; MS $\mathrm{AP}(-) m / z 481.96(\mathrm{M}-\mathrm{H})^{-}$.

\section{5-[(1,3-Diphenyl-1H-pyrazol-4-yl)methylene]-4-oxo-2-thioxo-3-}

thiazolidinepropanoic acid (8). mp $232-234{ }^{\circ} \mathrm{C} ;{ }^{1} \mathrm{H} \mathrm{NMR}\left(300 \mathrm{MHz}, \mathrm{CDCl}_{3}\right) \delta 2.49$

$(\mathrm{t}, 2 \mathrm{H}, J=8.1), 4.17(\mathrm{t}, 2 \mathrm{H}, J=7.8), 7.16-7.22(\mathrm{~m}, 1 \mathrm{H}), 7.26-7.36(\mathrm{~m}, 5 \mathrm{H}), 7.44(\mathrm{~d}, 2 \mathrm{H}, J$ $=7.5), 7.51(\mathrm{~s}, 1 \mathrm{H}), 7.52(\mathrm{~d}, 2 \mathrm{H}, J=1.2), 8.06(\mathrm{~s}, 1 \mathrm{H}) ; \mathrm{MS} \mathrm{AP}(-) \mathrm{m} / \mathrm{z} 434.00(\mathrm{M}-\mathrm{H})^{-}$. 
JM049222O - Supporting Information

5-[[3-(4-Methylphenyl)-1-phenyl-1H-pyrazol-4-yl]methylene]-4-oxo-2-thioxo-3thiazolidineacetic acid (9). mp $271-272{ }^{\circ} \mathrm{C} ;{ }^{1} \mathrm{H}$ NMR (300 MHz, DMSO) $\delta 2.41$ (s, $3 \mathrm{H}), 4.51(\mathrm{~s}, 2 \mathrm{H}), 7.34-7.47(\mathrm{~m}, 3 \mathrm{H}), 7.64-7.49(\mathrm{~m}, 5 \mathrm{H}), 8.07(\mathrm{~d}, 2 \mathrm{H}, J=8.4), 8.81(\mathrm{~s}$, 1H); $\mathrm{MS} \mathrm{AP(-)} \mathrm{m/z} 435.01(\mathrm{M}-\mathrm{H})^{-}$.

\section{5-[[5-(3,4-Dichlorophenyl)-2-furanyl]methylene]-4-oxo-2-thioxo-3-}

thiazolidinehexanoic acid (10). $\mathrm{mp} 182-188^{\circ} \mathrm{C} ;{ }^{1} \mathrm{H} \mathrm{NMR}\left(300 \mathrm{MHz}, \mathrm{CDCl}_{3}\right) \delta 0.94-$ $1.07(\mathrm{~m}, 2 \mathrm{H}), 1.19-1.36(\mathrm{~m}, 4 \mathrm{H}), 1.85(\mathrm{t}, 2 \mathrm{H}, J=7.6), 3.68(\mathrm{t}, 2 \mathrm{H}, J=7.5), 6.63-6.67(\mathrm{~m}$, 2H), $7.10(\mathrm{~s}, 1 \mathrm{H}), 7.16-7.28(\mathrm{~m}, 2 \mathrm{H}),, 7.45(\mathrm{~d}, 1 \mathrm{H}, J=2.2) ; \quad \operatorname{MS} \operatorname{AP}(-) \mathrm{m} / \mathrm{z} 468.70(\mathrm{M}-$ $\mathrm{H})^{-}$.

5-(1H-Indol-3-ylmethylene)-4-oxo-2-thioxo-3-thiazolidinehexanoic acid (11). mp 203-205 ${ }^{\circ} \mathrm{C} ;{ }^{1} \mathrm{H}$ NMR (300 MHz, DMSO) $\delta$ 1.26-1.38 (m, 2H), 1.48-1.59 (m, 2H), $1.60-$ $1.67(\mathrm{~m}, 2 \mathrm{H}), 2.22(\mathrm{t}, 2 \mathrm{H}, J=7.3), 4.02(\mathrm{t}, 2 \mathrm{H}, J=7.4), 7.20-7.31(\mathrm{~m}, 2 \mathrm{H}), 7.52(\mathrm{~d}, 1 \mathrm{H}, J$ $=7.1), 7.89(\mathrm{~s}, 1 \mathrm{H}), 7.96(\mathrm{~d}, 1 \mathrm{H}, J=7.3), 8.08(\mathrm{~s}, 1 \mathrm{H}) ; \quad \operatorname{MS~AP}(+) \mathrm{m} / \mathrm{z} 374.91(\mathrm{M}+\mathrm{H})^{+}$, MS AP(-) m/z $373.97(\mathrm{M}-\mathrm{H})^{-}$.

5-[(3-Ethoxy-4-hydroxyphenyl)methylene]-4-oxo-2-thioxo-3-thiazolidinehexanoic acid (12). $\mathrm{mp} 140-143{ }^{\circ} \mathrm{C} ;{ }^{1} \mathrm{H} \operatorname{NMR}\left(300 \mathrm{MHz}, \mathrm{CDCl}_{3}\right) \delta 1.11-1.82(\mathrm{~m}, 2 \mathrm{H}), 1.2(\mathrm{t}, 3 \mathrm{H}$, $J=7.2), 1.34-1.45(\mathrm{~m}, 4 \mathrm{H}), 1.98(\mathrm{t}, 2 \mathrm{H}, J=7.5), 3.78-3.89(\mathrm{~m}, 4 \mathrm{H}), 6.67-6.74(\mathrm{~m}, 3 \mathrm{H})$, 7.35 (s, 1H); MS AP(-) m/z $394.02(\mathrm{M}-\mathrm{H})^{-}$. 
JM049222O - Supporting Information

5-[(2-Methoxyphenyl)methylene]-4-oxo-2-thioxo-3-thiazolidinehexanoic acid (13). mp $114-116{ }^{\circ} \mathrm{C} ;{ }^{1} \mathrm{H}$ NMR $\left(300 \mathrm{MHz}, \mathrm{CDCl}_{3}\right) \delta$ 1.36-1.52 (m, 2H), 1.64-1.82 (m, 4H), $2.37(\mathrm{t}, 2 \mathrm{H}, J=7.3), 3.92(\mathrm{~s}, 3 \mathrm{H}), 4.12(\mathrm{t}, 2 \mathrm{H}, J=7.3), 6.95(\mathrm{~d}, 1 \mathrm{H}, J=7.8), 7.00-7.08$ (m, 1H), 7.37-7.47 (m, 2H), $8.09(\mathrm{~s}, 1 \mathrm{H}) ; \quad \mathrm{MS} \mathrm{AP}(+) \mathrm{m} / \mathrm{z} 366.03(\mathrm{M}+\mathrm{H})^{+}$.

5-[(4-Fluorophenyl)methylene]-4-oxo-2-thioxo-3-thiazolidinehexanoic acid (14). mp 160-161 ${ }^{\circ} \mathrm{C} ;{ }^{1} \mathrm{H}$ NMR (300 MHz, DMSO) $\delta 1.23-1.34(\mathrm{~m}, 2 \mathrm{H}), 1.43-1.72(\mathrm{~m}, 4 \mathrm{H}), 2.18$ $(\mathrm{t}, 2 \mathrm{H}, J=7.2), 3.98(\mathrm{t}, 2 \mathrm{H}, J=7.1), 7.33-7.41(\mathrm{~m}, 2 \mathrm{H}), 7.64-7.72(\mathrm{~m}, 2 \mathrm{H}), 7.79(\mathrm{~s}, 1 \mathrm{H})$; MS AP(-) m/z $352.98(\mathrm{M}-\mathrm{H})^{-}$.

5-[(3-Bromo-4-methoxyphenyl)methylene]-4-oxo-2-thioxo-3-thiazolidinehexanoic acid (15). mp $179-181{ }^{\circ} \mathrm{C} ;{ }^{1} \mathrm{H} \mathrm{NMR}\left(300 \mathrm{MHz}, \mathrm{CDCl}_{3}\right) \delta 1.36-1.48(\mathrm{~m}, 2 \mathrm{H}), 1.62-1.79$ (m, 4H), $2.30(\mathrm{t}, 2 \mathrm{H}, J=8.1), 3.97(\mathrm{~s}, 3 \mathrm{H}), 4.11(\mathrm{t}, 2 \mathrm{H}, J=7.7), 7.02(\mathrm{~d}, 1 \mathrm{H}, J=8.6)$, 7.44-7.48 (m, 1H), $7.60(\mathrm{~s}, 1 \mathrm{H}), 7.70(\mathrm{~s}, 1 \mathrm{H})$; $\mathrm{MS} \mathrm{AP}(-) \mathrm{m} / \mathrm{z} 444.52(\mathrm{M}-\mathrm{H})^{-}$.

5-(2-Methyl-3-phenyl-2-propenylidene)-4-oxo-2-thioxo-3-thiazolidinehexanoic acid (16). mp 155-157 ${ }^{\circ} \mathrm{C} ;{ }^{1} \mathrm{H}$ NMR (300 MHz, DMSO) $\delta 1.23-1.37$ (m, 2H), 1.46-1.70 (m, 4H), 2.18-2.29 (m, 5H), $4.00(\mathrm{t}, 2 \mathrm{H}, J=7.5), 7.30-7.48(\mathrm{~m}, 6 \mathrm{H}), 7.55(\mathrm{~s}, 1 \mathrm{H}) ; \quad \mathrm{MS}$ $\mathrm{AP}(-) m / z 375.01(\mathrm{M}-\mathrm{H})^{-}$. 
JM049222O - Supporting Information

Soybean PDF2 cloning, expression, and purification. Based on the sequence similarity to Arabidopsis PDF2 gene, we identified soybean expressed-sequence-tagged (EST) clones from the corporate DNA database. Soybean PDF2 gene (matured form) was amplified from an EST cDNA clone with two PCR primers: 5'-

CCGTAAACCCTCCTCGAACCG-3' the sequence just preceding a NCoI site in the soybean PDF2 gene and a primer corresponding to the vector sequence with a BamHI site. The PCR product was inserted into the bacterial expression vector $\mathrm{pBX} 3$ (a modified form of $\mathrm{pET}$ vector) using NcoI and BamHI sites. The PDF2 gene sequence was confirmed by the corporate DNA sequencing facility. Soybean PDF2 genes were expressed and purified as described previously. ${ }^{2}$

PDF Inhibition Assay. Enzyme activity was measured in half-area 96-well microtiter plates using the PDF/FDH coupled spectrophotometric assay. ${ }^{3}$ The reaction mixture contained $50 \mathrm{mM}$ Hepes buffer (pH 7.2), 0.5 unit/ml FDH, $4 \mathrm{mM} \mathrm{NAD}^{+}$, and PDF. Test compounds in DMSO (final concentration of 4\%) were pre-incubated with the enzyme in reaction mix for 10 min prior to adding the substrate, for-Met-Ala-Ser (final concentration of $2 \mathrm{mM}$ ). The reaction was monitored by following the conversion of $\mathrm{NAD}^{+}$to $\mathrm{NADH}$ at $340 \mathrm{~nm}$. Inhibition was determined based on the reaction rates obtained over a 10 min period.

The effect of varying substrate and inhibitor concentration on enzyme kinetics was monitored to determine the type of inhibition. The results were analyzed using the GraFit software package (Erithacus Software Limited). The lines in figure 2 were generated using a $\mathrm{K}_{\mathrm{m}}$ value of $6.4 \mathrm{mM}$. 
JM049222O - Supporting Information

Virtual Ligand Screening: additional details. The database used in our virtual screening was compiled from the "off the shelf" compounds from various vendors. Structures of those compounds were cleaned and tautomer state checked. A set of structure exclusion queries were applied to remove reactive and potentially toxic compounds and well investigated areas of chemistry. The remaining structures were combined into a single database.

Two independent ICM VLS runs were performed and lowest score of the two runs assigned to each compound. To explore the feasibility of discovering non-chelating inhibitors, an additional VLS was carried out with two crystallographic water molecules present (a metal bound water and a nearby water) in the active site.

To remove some of the artifacts of VLS, two simple filters were applied: (1) only compounds with at least one heavy atom within $3.5 \AA$ from the metal ion were retained, since an interaction of the ligand with the active site metal was expected; and (2) compounds which had heavy atom clashes with receptor shorter than 0.8 of the sum of the Van der Waals radii were removed. After filtering, a list of 4526 candidate compounds was selected. 
JM049222O - Supporting Information

\section{References for Supporting Information}

(1) Bogolyubskaya, L. T.; Bogolyubskii, V. A.; Sytnik, Z. P. 3-(ع-

Carboxyamyl)rhodanine and some of its derivatives. J. Org. Chem. USSR

(Engl.Transl.) 1966, 2, 1311-1313.

(2) Zheng, Y.J., Tao, Y., Zhang, W., and Jordan D. B. Inhibition of Fungal Aldose

Reductase. Protein and Peptide Lett. 2001, 8, 407-412.

(3) Lazennec, C. and Meinnel, T. Formate Dehydrogenase-Coupled Spectrophotometric Assay of Peptide Deformylase. Anal. Biochem. 1997, 244, 180-182. 